\title{
3
}

\section{Coevolution of the Cell Cycle and Deferred-Use Cells}

Siim Pauklin

University of Oxford

\subsection{The Connection of Cell Cycle and Cell Fate Decisions in Stem Cells}

The cell cycle and cell fate decisions are interlinked in a broad range of developmental contexts in many organisms. Coordination of stem cell proliferation and differentiation is essential for normal development, organ homeostasis, and tissue repair through a direct interplay between cell cycle progression and differentiation in somatic stem cells in the skin, brain, gut, and hematopoietic system (Fuchs 2009; Lange and Calegari 2010; Li and Clevers 2010). Particularly important insight to the processes coordinating cell fate and the cell cycle has been derived from pluripotent stem cells. Research in the past decades has shown that different cell cycle phases and mitosis provide intricate layers for regulating stem cell self-renewal, exit from the stem cell state, and guide stem cell differentiation to various cellular identities (Soufi and Dalton 2016). These processes involve coupling of the cell cycle with distinct gene expression programs via molecular processes mediated by transcription factors, cell type-specific patterns of epigenetic modifications, and chromatin organization. Figure 3.1 depicts the coordination of cell cycle and cell fate decisions.

$<$ COMP: Place Figure 3.1 Here>

The cell cycle represents a sequence of events in which chromosomes are replicated during DNA synthesis in $\mathrm{S}$ phase and segregated to the arising daughter cells via mitosis in $\mathrm{M}$ phase. These key events are separated by gap phases, G1 and G2, which control the correct timing and order 
of cellular events. The gap phase known as G1 starts the cell cycle after cell division and marks the stage when the arising cell either continues proliferation and prepares for DNA replication, or exits from the cell cycle and enters a nonproliferative stage known as G0 phase. The gap phase known as G2 follows DNA synthesis in S phase and represents a stage when the cell prepares for the subsequent cell division into two daughter cells.

Thus, the cell cycle of a continuously proliferating cell can be divided into G1, S, G2, and M phases, which will be repeated upon cell division. These successive cell cycle stages are regulated by the activity of cyclins and cyclin-dependent kinases (CDKs) that phosphorylate intracellular proteins essential for the different cycle phases and their ordered progression. A central decision stage for the exit or continuation for the cell cycle, known as the restriction (R-) point, is made in the G1 phase and regulated by CDK-mediated phosphorylation of retinoblastoma (RB) family proteins (Blagosklonny and Pardee 2002). This mechanism, which governs the growth and proliferation of most cell types, has an important role in stem cell selfrenewal, differentiation, and quiescence in a broad range of developmental contexts (Tetteh et al. 2015; Li and Clevers 2010).

A cell needs to reach sufficient volume in G1 phase before progressing to S phase and committing to another round of cell division (Ginzberg et al. 2015). This cell size-associated mechanism is widely conserved in evolution, for instance, budding yeast have a size-regulated restriction point in G1 phase called "Start" that resembles the R-point in mammals (Jorgensen and Tyers 2004). In addition to cell size, the R-point connects cell cycle decisions to cell fate decisions via external growth-promoting signals. In the presence of such mitogenic signals, normal cells progress through the restriction point and enter $\mathrm{S}$ phase due to the activation of genes necessary for cell cycle progression. On the other hand, when mitogenic signals are absent, 
the cells will neither enter S phase nor replicate their DNA. In pluripotent stem cells, exit from the cell cycle due to MYC proto-oncogene depletion leads to a developmental state that resembles the diapause stage (Scognamiglio et al. 2016). In addition to regulating stem cells, deregulation of the G1 checkpoint mechanism plays an important role in tumorigenesis, since the cells will not be able to block cell cycle progression upon environmental stress or the absence of mitogenic signals.

\subsection{Coordination of Cell Cycle and Cell Fate Decisions is Present in Many Species}

The connection between cell cycle and cell fate decisions is present across the whole evolutionary tree. For instance, haploid budding yeast switch between two alleles of the matingtype locus via the $\mathrm{HO}$ endonuclease mediated recombination event in $\mathrm{G} 1$ phase, which leads to cells that can mate and form diploid cells (Thon et al. 2018). The amoeba Dictyostelium enters a prespore identity upon nutrient starvation conditions in G1 phase while undertaking a prestalk identity when the signal is received in S and G2 phases (Gomer and Firtel 1987).

Cell cycle and cell fate decisions are also interconnected in multicellular organisms, spanning the nematode Caenorhabditis elegans (Ambros 1999), zebrafish (Bouldin and Kimelman 2014), frogs, and sea squirts (Ogura et al. 2011). This interconnection also plays an important role in different tissues during mammalian embryogenesis. During neocortical development, murine cortical progenitor cells initiate different cell fate decisions when cells undergo cycling compared to when they are not cycling (McConnell and Kaznowski 1991). Murine fetal erythropoiesis requires $\mathrm{S}$ phase to induce the erythroid differentiation program via a GATA1dependent transcriptional regulation (Pop et al. 2010). Endocrine progenitors during pancreatic development initiate distinct cell fate decision depending on stage of the cell cycle when the cells 
receive differentiation signals: early G1 phase cells undergo an asymmetric cell division, whereas cells in late G1 phase progress through the cell cycle and give rise to two differentiated endocrine daughter cells (Kim et al. 2015).

While many events in multipotential stem cells are linked to their G1 phase, other cell cycle phases also can impact cell fate choice. For instance, a G2 phase transitioning time of Drosophila cells during bristle patterning is linked to cell fate decisions between a microchaete or a neural fate: a process known as lateral inhibition causes cells with elevated Notch signaling to divide first, compared to cells with lower Notch signaling, which extend their G2 phase and delay daughter cell production (Hunter et al. 2016).

\subsection{The Molecular Mechanisms Coordinating the Cell Cycle with Stem Cell Self-Renewal and Differentiation}

The correct balance in proliferation and differentiation of stem cells is essential for embryogenesis as well as for the formation and repair of adult tissues (Oshimori and Fuchs 2012; Coronado et al. 2013; Sela et al. 2012; Calder et al. 2012; Singh et al. 2013, 2015; Chazaud et al. 2006; Pauklin and Vallier 2015).

Human embryonic stem cells (hESCs) have an interconnection between cell cycle, self-renewal, and differentiation (Pauklin and Vallier 2015), while they exert a metastable state with heterogeneity at the single cell level. Metastability is a central feature of many types of stem cells and seems to be generally connected to cell cycle progression (Pauklin and Vallier 2013). Accordingly, hESCs are characterized by a specific cell cycle profile with a short G1 phase, which restricts entry into lineage commitment and maintains stem cell self-renewal (Pauklin and Vallier 2013; Singh et al. 2012, 2015); differentiation is associated with an increase in G1 phase length (Calder et al. 2012; Coronado et al. 2013). 
Pluripotent stem cells at the preimplantation developmental stage and cells maintained in vitro proliferate rapidly due to a truncated G1 phase and a shortened delay in S phase initiation. This shortened G1 phase supports the maintenance of pluripotency because cells reside for a briefer time period in the G1 cell cycle phase where cells are more prone to differentiation in response to external stimuli. When differentiation is initiated, elongation of the G1 phase makes cells more susceptible to irreversible germ layer commitment (Neganova et al. 2009; Pauklin and Vallier 2013; Boward et al. 2016; Ruiz et al. 2011). This cell cycle-mediated process results in a cell cycle-dependent capacity of differentiation in which hESCs initiate differentiation into the three germ layers during the transition from early to late G1 phase, while the S phase and G2/M primarily support pluripotency over differentiation (Gonzales et al. 2015; Pauklin and Vallier 2013). These mechanisms are governed by the cell cycle regulators cyclin D1-D3 that control differentiation signals TGF $\beta /$ Activin/Nodal-Smad2/3 pathway. hESCs in early G1 phase can only initiate differentiation into endoderm, whereas hESCs in late G1 are limited to neuroectoderm differentiation. The activity of Activin/Nodal signaling during cell cycle progression is controlled by cyclin D proteins that activate CDK4/6 and lead to the phosphorylation of Smad2 and Smad3 in their linker region. This mechanism blocks Smad2/3 shuttling in the nucleus in late G1, thereby preventing endoderm and allowing neuroectoderm specification (Pauklin and Vallier 2013). The G1 phase master regulators cyclin D1-D3 proteins also control mammalian stem cell differentiation and lineage specification on chromatin as transcription factors by blocking the expression of endoderm and mesoderm genes through transcriptional corepressors and inducing the transcription of neuroectoderm genes through transcriptional coactivators (Pauklin et al. 2016). 
Bivalent domains consisting of $\mathrm{H} 3 \mathrm{~K} 4 \mathrm{me} 3$ and $\mathrm{H} 3 \mathrm{~K} 27$ me3 modifications are enriched on developmental genes in G1 phase in pluripotent stem cells, and there is a particular increase in H3K4me3 marks in G1 phase (Singh et al. 2012, 2015). On the other hand, the H3K27me3 mark remains relatively stable on developmental genes throughout the cell cycle (Singh et al. 2012, 2015). This is consistent with the observation that developmental genes are primed for activation every time they go through G1 phase but are not activated unless the appropriate signaling networks are also active. G1-specific epigenetic changes at developmental genes also coincide with the establishment of DNA loops that bridge distal enhancers with proximal promoters. Altogether, this indicates that developmental genes in G1 phase are in a lineage-primed state due to changes in epigenetic landscapes and chromosome architectures, likely to be mediated via a crosstalk with transcription factors and CDKs.

$\mathrm{S}$ and G2 phases attenuate exit from a pluripotent state, because hESCs in these cell cycle phases possess an intrinsic propensity toward maintaining their pluripotent state. Among the components regulating this process are DNA damage checkpoint factors ATM/ATR-CHEK2p53 and cyclin B1 pathways (Gonzales et al. 2015). ATR/ATM enhances the activity of the TGF $\beta /$ Activin/Nodal pathway through p53 during S and G2. The upregulation of TGF $\beta$ then delays the decrease in the expression of pluripotency markers. This suggests that induction of hESC differentiation occurs during the G1 and M phases, while loss of pluripotency is achieved subsequently in S and G2 phases. Figure 3.2 depicts the connection of distinct phases of the cell cycle and pathways involved in regulating cell fate decisions and the exit from pluripotency in hESCs.

$<$ COMP: Place Figure 3.2 Here $>$

\subsection{Cell Division, Epigenetic Memory, and Mitotic Bookmarking}


Mitosis is the process of cell division that gives rise to two daughter cells from a single cell. Among the main characteristics of mitosis are (i) CDK-controlled processes of chromosome condensation and (ii) nuclear envelope breakdown (Spencer et al. 2000). During mitosis, the majority of factors regulating transcription-including developmental transcription factors, epigenetic "writers," and "readers" that deposit and interact with histone modificationsdissociate from chromatin, and cell type-specific transcription programs stop temporarily (Egli et al. 2008; Kellum et al. 1995; Minc et al. 1999; Voncken et al. 1999; McManus et al. 2006; Singh et al. 2015). This is accompanied by the removal of histone modifications (such as by histone acetylation) responsible for promoting gene expression; residual acetylations on distinct gene promoters are retained for "bookmarking” these loci throughout mitosis (Zhao et al. 2011). However, some repressive marks such as $\mathrm{H} 3 \mathrm{~K} 27 \mathrm{me} 3$ and $\mathrm{H} 3 \mathrm{~K} 9 \mathrm{me} 3$ remain on various loci. While mitosis is accompanied by a widespread erasure of the epigenome, bookmarking of some loci through cell division allows the arising daughter cells to restore previous gene expression programs (Zaidi et al. 2010). This process, which involves the reassembly of transcription complexes on enhancer and promoter regions, avoids the heterochromatinization of previously activated DNA loci, allowing for rapid reactivation of genes in G1 phase. Hence, mitotic bookmarking is important for maintaining the epigenetic memory throughout cell divisions and for interconnecting cell fate decisions with cell cycle-dependent epigenetic processes.

\subsection{Cell Cycle Regulation and Terminal Differentiation}

\subsubsection{Cyclin-Dependent Kinases and Cyclin-Dependent Kinase Inhibitors}

The cell cycle plays an important role in cell fate decisions by allowing the self-renewal of stem cells or progenitors while restricting further cell divisions during terminal differentiation. 
Differentiation is often associated with a change in cell cycle length (Coronado et al. 2013;

Savatier et al. 1996), suggesting that mechanisms controlling cell cycle length and progression could be involved in cell fate decisions. Proliferation of stem cells or progenitors maintains their developmentally plastic and immature cell identity, while terminal differentiation of cells during developmental processes usually requires the exit of cells from cell cycle in G1 phase.

The progression of cell cycle in mammalian cells is primarily controlled by cyclins and CDKs, which affect key transcriptional regulators of the RB tumor suppressor protein family. The activity of the cyclin $\mathrm{D} / \mathrm{CDK}$ complexes and thus cell proliferation is constrained by cyclindependent kinase inhibitors (CDKIs), which are subdivided into two families. The INK4 proteins (p16INK4a, p15INK4b, p18INK4c, and p19INK4d) bind to CDK4 and CDK6, and inhibit their kinase activities by interfering with their association with cyclin D proteins, while the Kip/Cip proteins (p21Cip1, p27Kip1, and p57Kip2) inhibit cyclin E-CDK2 (Sherr and Roberts 1999). Upregulation of CDKIs leads to the inhibition of CDKs during G1 phase and the hypophosphorylation of the RB protein family. This, in turn, allows RBs to bind to E2F transcription factors and thereby repress $\mathrm{E} 2 \mathrm{~F}$ target genes that are required for transitioning to $\mathrm{S}$ phase. The cyclin D-CDK4/6 complex can also bind to Kip/Cip CDKIs. However, this interaction enhances cyclin D-CDK4/6 activity since proteins such as p27 appear to limit INK4 CKIs' capacity to bind this complex. Hence, a complex combination of INK4 and KIP/CIP protein regulations determines cell cycle progression.

It was long believed that the cell cycle has only a passive role in cell fate decisions as a consequence of differentiation rather than being an active determinant guiding cell identity. Studies in various cell types have clarified this notion; for instance, neuronal differentiation is regulated by cell cycle stage-specific phosphorylation of the master transcription factors Ascll 
and Ngn2, while terminal differentiation of myoblasts is blocked by forced expression of cell proliferation-promoting factors such as cyclin D1 (Rao and Kohtz 1995) and by regulating the activity of MyoD1 during the cell cycle (Kitzmann and Fernandez 2001); terminal differentiation of myoblasts involves an upregulation of cell cycle inhibitor $\mathrm{p} 21$ by MyoD1 (Ruijtenberg and van den Heuvel 2015; Halevy et al. 1995). This suggests that cell cycle regulators have an active function in early events of differentiation by controlling the activity of master transcription factors and extracellular signals.

\subsubsection{Retinoblastoma Family Proteins}

The RB family of tumor suppressors (RBs: pRb, Rbl1 and Rbl2) are pivotal for cell cycle progression in mammalian cells. They control the G1 to S phase transition by reducing the transcriptional activity of E2F proteins (E2Fs), thereby leading to transcriptional repression of target genes necessary for proliferation. In turn, phosphorylation of RBs by cyclin D/CDK4-6 blocks interactions with E2Fs, permitting the induction of E2F-mediated transcription (Fiorentino et al. 2009). Besides their tumor suppressive function, RBs also are central for early mammalian development. Genetic studies in mice have shown that absence of $\mathrm{pRb}$ is embryonic lethal between E13 and E15 of gestation due to abnormal hematopoietic, neuronal, and eye lens development provoked by defects in cellular differentiation (Jacks et al. 1992; Korenjak and Brehm 2005).

$\mathrm{pRb}$ seems to direct differentiation of various cell types by controlling the activity of master regulators of differentiation. In contrast, the function of the other members of the RB family in differentiation is less well established. $\mathrm{Rbl1}^{-/-}$mice are viable and fertile but have impaired growth and exert myeloid hyperplasia (LeCouter et al. 1998a). More strikingly, Rbl2 ${ }^{-{ }^{--}}$embryos die between E11 and E13 due to disorganization in neural and dermatomyotomal structures 
(LeCouter et al. 1998b). Thus, Rb11/2 are likely to have a function in cell fate decision, which might not be directly overlapping with $\mathrm{pRb}$.

Targeted disruption of the three Rb-related genes in mESCs strongly alters their capacity of differentiation, while absence of RB protein function in hESCs induces cell death (Conklin et al. 2012). Moreover, mouse embryonic fibroblasts with a knockout for the three RB genes display a loss of G1 control and cellular immortalization (Sage et al. 2000; Dannenberg et al. 2000) that can be regarded as pathological self-renewal and that resembles the physiological self-renewal processes observed in embryonic or adult tissue-specific stem cells (Goding et al. 2014). In line with its function in controlling the self-renewal properties of stem cells or progenitors, $\mathrm{pRb}$ can restrict reprogramming and tumorigenesis by inhibiting pluripotency networks (Kareta et al. 2015). Considered together, these studies suggest a central function for RBs in cell fate decisions of stem cells and progenitors during embryonic development and in adult organs.

\subsection{Conclusions}

The cell cycle is tightly intertwined with cell fate decisions in diverse species ranging from yeast to human. Pluripotent stem cells in human have a cell cycle phase-specific responsiveness to differentiation signals, which changes from proneness to specification toward endoderm in early G1 phase and, in contrast, toward neuroectoderm in late G1 phase. The rest of the cell cycle is directed toward retaining the pluripotent identity of the stem cell. Together with lineage priming in G1 phase, the pluripotency network is prone to dissolution at this particular cell cycle phase. The rapid cycling of pluripotent stem cells with a truncated G1 phase and spending most of the time in S and G2 phases reduces the possibility for pluripotent stem cells to commit to lineage specification. During cell differentiation, there are large changes in the cell cycle profiles, which coordinate molecular complexes involving transcription factors, epigenetic modifying enzymes, 
epigenetic landscapes, and chromatic architecture. These cell cycle-orchestrated events ultimately govern gene expression programs unique to differentiated cell types.

Figure 3.1 The cell cycle regulates developmental processes. Crosstalk between cell cycle control and cell fate specification involves developmental signals and cyclin-dependent protein kinases (CDKs) that target transcription factors that control expression of developmental genes via epigenetic mechanisms. In addition, CDKs modulate the epigenetic landscape and chromosome architecture around developmental genes. Induction of target genes determines important cell fate decisions and subsequent lineage commitment. Abbreviations: bHLH, basic helix-loop-helix; ERK/MAPK, mitogen-activated protein kinases/extracellular signal-regulated kinases; SMAD, mothers against decapentaplegic homolog; TGF $\beta$, transforming growth factor beta.

Figure 3.2 Cell fate specification and the cell cycle are interlinked in stem cells. (a) Human embryonic stem cells have a short G1 phase that promotes the pluripotent state and allows rapid proliferation. (b) Dissolution of pluripotency and lineage priming work in concert to orchestrate exit from pluripotency and initiate cell fate decisions in G1 phase. Cell fate specification of human embryonic stem cells (hESCs) starts in the G1 phase upon receiving differentiation signals and is regulated by a crosstalk between cyclin D-CDK4/6 and TGF $\beta$-Smad2/3 pathway. (c) Cell fate commitment is further achieved in G2/M when exit from pluripotency is mediated through cell cycle-dependent mechanisms that are governed by cyclin E and ATR/ATM-Chk2p53 pathway, as well as mitotic bookmarking. The daughter cells that arise after cell division either maintain their pluripotent state upon signals that support stem cell self-renewal or undergo differentiation to a developmentally restricted cell identity. Abbreviations: ATR/ATM, ataxia 
telangiectasia and Rad3-related protein/ataxia telangiectasia mutated; CDK4/6, cyclin-dependent kinase 4/6; Chk2, checkpoint kinase 2; p53, tumor protein p53.

\section{References}

Ambros, V. 1999. Cell cycle-dependent sequencing of cell fate decisions in Caenorhabditis

elegans vulva precursor cells. Development 126:1947-1956.

Blagosklonny, M. V., and Pardee, A. B. 2002. The restriction point of the cell cycle. Cell Cycle

1:103-110.

Bouldin, C. M., and Kimelman, D. 2014. Cdc25 and the importance of G2 control: insights from

developmental biology. Cell Cycle 13:2165-2171. doi:10.4161/cc.29537.

Boward, B., Wu, T., and Dalton, S. 2016. Concise review: control of cell fate through cell cycle

and pluripotency networks. Stem Cells 34:1427-1436. doi:10.1002/stem.2345.

Calder, A., Roth-Albin, I., Bhatia, S., Pilquil, C., Lee, J. H., Bhatia, M., Levadoux-Martin, M.,

McNicol, J., Russell, J., Collins, T., and Draper, J. S. 2012. Lengthened G1 phase indicates

differentiation status in human embryonic stem cells. Stem Cells Dev. 22:279-295.

doi:10.1089/scd.2012.0168. 
Chazaud, C., Yamanaka, Y., Pawson, T., and Rossant, J. 2006. Early lineage segregation

between epiblast and primitive endoderm in mouse blastocysts through the Grb2-MAPK

pathway. Dev. Cell 10:615-624. doi:10.1016/j.devcel.2006.02.020.

Conklin, J. F., Baker, J., and Sage, J. 2012. The RB family is required for the self-renewal and

survival of human embryonic stem cells. Nat. Commun. 3:1244. doi:10.1038/ncomms2254.

Coronado, D., Godet, M., Bourillot, P. Y., Tapponnier, Y., Bernat, A., Petit, M., Fanassieff, M.,

Markossian, S., Malashicheva, A., Iacone, R., Anastassiadis, K., and Savatier, P. 2013. A

short G1 phase is an intrinsic determinant of naive embryonic stem cell pluripotency. Stem

Cell Res. 10:118-131. doi:10.1016/j.scr.2012.10.004.

Dannenberg, J. H., van Rossum, A., Schuijff, L., and te Riele, H. 2000. Ablation of the

retinoblastoma gene family deregulates $\mathrm{G}(1)$ control causing immortalization and increased

cell turnover under growth-restricting conditions. Genes Dev. 14:3051-3064.

Egli, D., Birkhoff, G., and Eggan, K. 2008. Mediators of reprogramming: transcription factors

and transitions through mitosis. Nat. Rev. Mol. Cell Biol. 9:505-516. doi:10.1038/nrm2439.

Fiorentino, F. P., Symonds, C. E., Macaluso, M., and Giordano, A. 2009. Senescence and

p130/Rb12: a new beginning to the end. Cell Res. 19:1044-1051. doi:10.1038/cr.2009.96. 
Fuchs, E. 2009. The tortoise and the hair: slow-cycling cells in the stem cell race. Cell 137:811-

819. doi:10.1016/j.cell.2009.05.002.

Ginzberg, M. B., Kafri, R., and Kirschner, M. 2015. Cell biology. On being the right (cell) size.

Science 348:1245075. doi:10.1126/science.1245075.

Goding, C. R., Pei, D., and Lu, X. 2014. Cancer: pathological nuclear reprogramming? Nat. Rev.

Cancer 14:568-573. doi:10.1038/nrc3781.

Gomer, R. H., and Firtel, R. A. 1987. Cell-autonomous determination of cell-type choice in

Dictyostelium development by cell-cycle phase. Science 237:758-762.

Gonzales, K. A., Liang, H., Lim, Y. S., Chan, Y. S., Yeo, J. C., Tan, C. P., Gao, B., Le, B., Tan,

Z. Y., Low, K. Y., Liou, Y. C., Bard, F., and Ng, H. H. 2015. Deterministic restriction on

pluripotent state dissolution by cell-cycle pathways. Cell 162:564-579.

doi:10.1016/j.cell.2015.07.001.

Halevy, O., Novitch, B. G., Spicer, D. B., Skapek, S. X., Rhee, J., Hannon, G. J., Beach, D., and

Lassar, A. B. 1995. Correlation of terminal cell cycle arrest of skeletal muscle with induction

of p21 by MyoD. Science 267:1018-1021. 
Hunter, G. L., Hadjivasiliou, Z., Bonin, H., He, L., Perrimon, N., Charras, G., and Baum, B.

2016. Coordinated control of Notch/Delta signalling and cell cycle progression drives lateral

inhibition-mediated tissue patterning. Development 143:2305-2310. doi:10.1242/dev.134213.

Jacks, T., Fazeli, A., Schmitt, E. M., Bronson, R. T., Goodell, M. A., and Weinberg, R. A. 1992.

Effects of an Rb mutation in the mouse. Nature 359:295-300. doi:10.1038/359295a0.

Jorgensen, P., and Tyers, M. 2004. How cells coordinate growth and division. Curr. Biol.

14:R1014-R1027. doi:10.1016/j.cub.2004.11.027.

Kareta, M. S., Gorges, L. L., Hafeez, S., Benayoun, B. A., Marro, S., Zmoos, A. F., Cecchini, M.

J., Spacek, D., Batista, L. F., O‘Brien, M., Ng, Y. H., Ang, C. E., Vaka, D., Artandi, S. E.,

Dick, F. A., Brunet, A., Sage, J., and Wernig, M. 2015. Inhibition of pluripotency networks

by the $\mathrm{Rb}$ tumor suppressor restricts reprogramming and tumorigenesis. Cell Stem Cell 16:39-

50. doi:10.1016/j.stem.2014.10.019.

Kellum, R., Raff, J. W., and Alberts, B. M. 1995. Heterochromatin protein 1 distribution during

development and during the cell cycle in Drosophila embryos. J. Cell Sci. 108:1407-1418. 
Kim, Y. H., Larsen, H. L., Ru, P., Lemaire, L. A., Ferrer, J., and Grapin-Botton, A. 2015. Cell

cycle-dependent differentiation dynamics balances growth and endocrine differentiation in the

pancreas. PLoS Biol. 13:e1002111. doi:10.1371/journal.pbio.1002111.

Kitzmann, M., and Fernandez, A. 2001. Crosstalk between cell cycle regulators and the

myogenic factor MyoD in skeletal myoblasts. Cell. Mol. Life Sci. 58:571-579.

Korenjak, M., and Brehm, A. 2005. E2F-Rb complexes regulating transcription of genes

important for differentiation and development. Curr. Opin. Genet. Dev. 15:520-527.

doi:10.1016/j.gde.2005.07.001.

Lange, C., and Calegari, F. 2010. Cdks and cyclins link G1 length and differentiation of

embryonic, neural and hematopoietic stem cells. Cell Cycle 9:1893-1900.

LeCouter, J. E., Kablar, B., Hardy, W. R., Ying, C., Megeney, L. A., May, L. L., and Rudnicki,

M. A. 1998a. Strain-dependent myeloid hyperplasia, growth deficiency, and accelerated cell

cycle in mice lacking the Rb-related p107 gene. Mol. Cell. Biol. 18:7455-7465.

LeCouter, J. E., Kablar, B., Whyte, P. F., Ying, C., and Rudnicki, M. A. 1998b. Strain-dependent

embryonic lethality in mice lacking the retinoblastoma-related p130 gene. Development

125:4669-4679. 
Li, L., and Clevers, H. 2010. Coexistence of quiescent and active adult stem cells in mammals.

Science 327:542-545. doi:10.1126/science.1180794.

McConnell, S. K., and Kaznowski, C. E. 1991. Cell cycle dependence of laminar determination

in developing neocortex. Science 254:282-285.

McManus, K. J., Biron, V. L., Heit, R., Underhill, D. A., and Hendzel, M. J. 2006. Dynamic

changes in histone $\mathrm{H} 3$ lysine 9 methylations: identification of a mitosis-specific function for

dynamic methylation in chromosome congression and segregation. J. Biol. Chem. 281:8888-

8897. doi:10.1074/jbc.M505323200.

Minc, E., Allory, Y., Worman, H. J., Courvalin, J. C., and Buendia, B. 1999. Localization and

phosphorylation of HP1 proteins during the cell cycle in mammalian cells. Chromosoma

108:220-234.

Neganova, I., Zhang, X., Atkinson, S., and Lako, M. 2009. Expression and functional analysis of

G1 to $\mathrm{S}$ regulatory components reveals an important role for CDK2 in cell cycle regulation in

human embryonic stem cells. Oncogene 28:20-30. doi:10.1038/onc.2008.358. 
Ogura, Y., Sakaue-Sawano, A., Nakagawa, M., Satoh, N., Miyawaki, A., and Sasakura, Y. 2011.

Coordination of mitosis and morphogenesis: role of a prolonged G2 phase during chordate

neurulation. Development 138:577-587. doi:10.1242/dev.053132.

Oshimori, N., and Fuchs, E. 2012. The harmonies played by TGF-beta in stem cell biology. Cell

Stem Cell 11:751-764. doi:10.1016/j.stem.2012.11.001.

Pauklin, S., Madrigal, P., Bertero, A., and Vallier, L. 2016. Initiation of stem cell differentiation

involves cell cycle-dependent regulation of developmental genes by Cyclin D. Genes Dev.

30:421-433. doi:10.1101/gad.271452.115.

Pauklin, S., and Vallier, L. 2013. The cell-cycle state of stem cells determines cell fate

propensity. Cell 155:135-47. doi:10.1016/j.cell.2013.08.031.

Pauklin, S., and Vallier, L. 2015. Activin/nodal signalling in stem cells. Development 142:607-

619. doi:10.1242/dev.091769.

Pop, R., Shearstone, J. R., Shen, Q., Liu, Y., Hallstrom, K., Koulnis, M., Gribnau, J., and

Socolovsky, M. 2010. A key commitment step in erythropoiesis is synchronized with the cell

cycle clock through mutual inhibition between PU.1 and S-phase progression. PLoS Biol.

8:e1000484. doi:10.1371/journal.pbio.1000484. 
Rao, S. S., and Kohtz, D. D. 1995. Positive and negative regulation of D-type cyclin expression

in skeletal myoblasts by basic fibroblast growth factor and transforming growth factor beta. A

role for cyclin D1 in control of myoblast differentiation. J. Biol. Chem. 270:4093-4100.

Ruijtenberg, S., and van den Heuvel, S. 2015. G1/S inhibitors and the SWI/SNF complex control

cell-cycle exit during muscle differentiation. Cell 162:300-313.

doi:10.1016/j.cell.2015.06.013.

Ruiz, S., Panopoulos, A. D., Herrerias, A., Bissig, K. D., Lutz, M., Berggren, W. T., Verma, I.

M., and Izpisua Belmonte, J. C. 2011. A high proliferation rate is required for cell

reprogramming and maintenance of human embryonic stem cell identity. Curr. Biol. 21:45-

52. doi:10.1016/j.cub.2010.11.049.

Sage, J., Mulligan, G. J., Attardi, L. D., Miller, A., Chen, S., Williams, B., Theodorou, E., and

Jacks, T. 2000. Targeted disruption of the three Rb-related genes leads to loss of G(1) control

and immortalization. Genes Dev. 14:3037-3050.

Savatier, P., Lapillonne, H., van Grunsven, L. A., Rudkin, B. B., and Samarut, J. 1996.

Withdrawal of differentiation inhibitory activity/leukemia inhibitory factor up-regulates D- 
type cyclins and cyclin-dependent kinase inhibitors in mouse embryonic stem cells. Oncogene

\section{2:309-322.}

Scognamiglio, R., Cabezas-Wallscheid, N., Thier, M. C., Altamura, S., Reyes, A., Prendergast,

A. M., Baumgartner, D., Carnevalli, L S., Atzberger, A., Haas, S., von Paleske, L., Boroviak,

T., Worsdorfer, P., Essers, M. A., Kloz, U., Eisenman, R. N., Edenhofer, F., Bertone, P.,

Huber, W., van der Hoeven, F., Smith, A., and Trumpp, A. 2016. Myc Depletion induces a

pluripotent dormant state mimicking diapause. Cell 164:668-680.

doi:10.1016/j.cell.2015.12.033.

Sela, Y., Molotski, N., Golan, S., Itskovitz-Eldor, J., and Soen, Y. 2012. Human embryonic stem

cells exhibit increased propensity to differentiate during the G1 phase prior to

phosphorylation of retinoblastoma protein. Stem Cells 30:1097-1108. doi:10.1002/stem.1078.

Sherr, C. J., and Roberts, J. M. 1999. CDK inhibitors: positive and negative regulators of G1-

phase progression. Genes Dev. 13:1501-1512.

Singh, A. M., Chappell, J., Trost, R., Lin, L., Wang, T., Tang, J., Matlock, B. K., Weller, K. P.,

Wu, H., Zhao, S., Jin, P., and Dalton, S. 2013. Cell-cycle control of developmentally 
regulated transcription factors accounts for heterogeneity in human pluripotent cells. Stem

Cell Rep. 1:532-544. doi:10.1016/j.stemcr.2013.10.009.

Singh, A. M., Reynolds, D., Cliff, T., Ohtsuka, S., Mattheyses, A. L., Sun, Y., Menendez, L.,

Kulik, M., and Dalton, S. 2012. Signaling network crosstalk in human pluripotent cells: a

Smad2/3-regulated switch that controls the balance between self-renewal and differentiation.

Cell Stem Cell 10:312-326. doi:10.1016/j.stem.2012.01.014.

Singh, A. M., Sun, Y., Li, L., Zhang, W., Wu, T., Zhao, S., Qin, Z., and Dalton, S. 2015. Cell-

cycle control of bivalent epigenetic domains regulates the exit from pluripotency. Stem Cell

Rep. 5:323-336. doi:10.1016/j.stemcr.2015.07.005.

Soufi, A., and Dalton, S. 2016. Cycling through developmental decisions: how cell cycle

dynamics control pluripotency, differentiation and reprogramming. Development 143:4301-

4311. doi:10.1242/dev.142075.

Spencer, C. A., Kruhlak, M. J., Jenkins, H. L., Sun, X., and Bazett-Jones, D. P. 2000. Mitotic

transcription repression in vivo in the absence of nucleosomal chromatin condensation. J. Cell

Biol. 150:13-26. 
Tetteh, P. W., Farin, H. F., and Clevers, H. 2015. Plasticity within stem cell hierarchies in mammalian epithelia. Trends Cell Biol. 25:100-108. doi:10.1016/j.tcb.2014.09.003.

Thon, G., Maki, T., Haber, J. E., and Iwasaki, H. 2018. Mating-type switching by homologydirected recombinational repair: a matter of choice. Curr. Genet. 65:351-362.

doi:10.1007/s00294-018-0900-2.

Voncken, J. W., Schweizer, D., Aagaard, L., Sattler, L., Jantsch, M. F., and van Lohuizen, M. 1999. Chromatin-association of the Polycomb group protein BMI1 is cell cycle-regulated and correlates with its phosphorylation status. J. Cell Sci. 112:4627-4639.

Zaidi, S. K., Young, D. W., Montecino, M. A., Lian, J. B., van Wijnen, A. J., Stein, J. L., and Stein, G. S. 2010. Mitotic bookmarking of genes: a novel dimension to epigenetic control.

Nat. Rev. Genet. 11:583-589. doi:10.1038/nrg2827.

Zhao, R., Nakamura, T., Fu, Y., Lazar, Z., and Spector, D. L. 2011. Gene bookmarking accelerates the kinetics of post-mitotic transcriptional re-activation. Nat. Cell. Biol. 13:12951304. doi: $10.1038 / \mathrm{ncb} 2341$. 